\title{
ON THE POSITIVE AND NEGATIVE SOLUTIONS OF LAPLACIAN BVP WITH NEUMANN BOUNDARY CONDITIONS
}

\author{
G. A. AFROUZI*, M. KHALEGHY MOGHADDAM, J. MOHAMMADPOUR AND M. \\ ZAMENI
}

\begin{abstract}
In this paper, we consider the following Neumann boundary value problem

$$
\left\{\begin{array}{l}
-u^{\prime \prime}(x)=u^{3}(x)-\lambda|u(x)|, \quad x \in(0,1), \\
u^{\prime}(0)=0=u^{\prime}(1)
\end{array}\right.
$$

where $\lambda \in \mathbb{R}$ is parameter. We study the positive and negative solutions of this problem with respect to a parameter $\rho$ (i.e. $u(0)=\rho$ ) in all $\mathbb{R}^{*}$. By using a quadrature method, we obtain our results. Also we provide some details about the solutions that are obtained.
\end{abstract}

\section{INTRODUCTION}

Consider the nonlinear two point boundary value problem

$$
\begin{aligned}
-u^{\prime \prime}(x) & =u^{3}(x)-\lambda|u(x)|, \quad x \in(0,1), \\
u^{\prime}(0) & =0=u^{\prime}(1),
\end{aligned}
$$

where $\lambda \in \mathbb{R}$ is parameter.

We study the positive and negative solutions of this problem with respect to a parameter $\rho$ (that is the value of the solutions at zero, i.e. $u(0)=\rho$ ). Also by using a quadrature method, we obtain our results. In [2] and [7] for semipositone problems with $p$-Laplacian operator, existence and multiplicity results have been established with Neumann boundary value conditions and Dirichlet boundary value conditions, respectively. In [5], for semipositone and positone problems have been studied by Anuradha, Maya and Shivaji by using a quadrature method with Neumann-Robin boundary conditions and Laplacian operator.

Date: Received: 07 March 2009; Revised: 17 April 2009.

* Corresponding author.

2000 Mathematics Subject Classification. 34B15, 34B18.

Key words and phrases. Positive and negative solutions; Interior critical points; Quadrature method; Neumann boundary condition; Laplacian problem. 
In [9] for semipositone problems, existence and multiplicity results have been established with Laplacian operator and Neumann boundary value conditions. Also, in [3], [6] and [8] for semipositone problems with Laplacian operator have been studied for solution curves with Dirichlet boundary value conditions.

This paper is organized as follows. In Section 2, we first state some remarks and then our main results and finally in Section 3, we provide the proof of our main results that contains several lemmas.

\section{Main Results}

By a solution of (1)-(2) we mean a function $u \in C^{1}([0,1])$ for which $u^{\prime} \in$ $C^{1}([0,1])$ and both the equation and the boundary value conditions are satisfied Remark 1 If $u$ is a solution to (1)-(2) at $\lambda$, then $-u$ is a solution to (1)-(2) at $-\lambda$.

Remark 2 Let $u$ is a solution to (1)-(2) at $\lambda$ then

$$
\int_{0}^{1} u^{3}(x) u^{\prime}(x) d x=\int_{0}^{1} \lambda|u(x)| u^{\prime}(x) d x .
$$

Remark 3 Every solution $u$ of (1)-(2) is symmetric about any interior critical points such that for any point $x_{0} \in(0,1)$ where $u^{\prime}\left(x_{0}\right)=0$, we have $u\left(x_{0}-z\right)=$ $u\left(x_{0}+z\right)$ for all $z \in\left[0, \min \left\{x_{0}, 1-x_{0}\right\}\right]$.

In fact, let $w_{1}(z)=u\left(x_{0}-z\right)$ and $w_{2}(z)=u\left(x_{0}+z\right)$, then it is clear that both $w_{1}$ and $w_{2}$ satisfy the IVP

$$
\left\{\begin{array}{l}
-w^{\prime \prime}(x)=w^{3}(x)-\lambda|w(x)| \\
w(0)=u\left(x_{0}\right) \\
w^{\prime}(0)=0
\end{array}\right.
$$

Hence, by uniqueness theorem for ODE, one can conclude result.

Remark 4 If $u$ is a solution to (1)-(2), then $u(1-x)$ is also a solution to (1)-(2). Definition 5 Let $u$ be a solution to problem (1)-(2) and $k$ be the number of interior critical points of $u$ then define the following sets:

$A_{k}=\{u: u$ is decreasing at the beginning and end of $(0,1)\}$,

$B_{k}=\{u: u$ is decreasing at the beginning and increasing at the end of $(0,1)\}$,

$C_{k}=\{u: u$ is increasing at the beginning and decreasing at the end of $(0,1)\}$,

$D_{k}=\{u: u$ is increasing at the beginning and end of $(0,1)\}$.

Theorem 1 Let $\rho \in \mathbb{R}$, then,

(a)

(1) the problem (1)-(2) has exactly one positive solution $u$ with $u(0)=\rho$ at any $\lambda \in S_{\rho}$ where

$$
S_{\rho}= \begin{cases}\left(0, \rho^{2}\right) \bigcup\left(\rho^{2},+\infty\right), & \rho>0 \\ (0, \infty), & \rho=0, \\ (0, \infty), & \rho<0,\end{cases}
$$

(2) the problem (1)-(2) has no positive solution with $u(0)=\rho$ at any $\lambda \in S_{\rho}^{c}$ 
(b) the corresponding solution is difined by

$$
\int_{\rho}^{u(x)}\{M(\rho, \lambda, s)\}^{-\frac{1}{2}} d s=\kappa\{2\}^{1 / 2} x, \quad x \in\left(0, x_{0}\right),
$$

such that

$$
\kappa=\left\{\begin{array}{l}
-, \quad \lambda \in\left(0, \rho^{2} / 2\right), \rho>0 \\
+, \quad \lambda \in(0, \infty), \rho=0 \\
+, \quad \lambda \in(0, \infty), \rho<0
\end{array}\right.
$$

and $k$ be the number of interior critical points of $u$ where $k=0,1,2, \ldots$ and if $k>0, x_{0}$ is the first interior critical point.

Theorem 2 Let $u$ be a solution to (1)-(2) at $\lambda \in S_{\rho}$ with $u(0)=\rho$ and $k$ be the number of interior critical points such that $k=0,1,2, \ldots$, then solution $u$

(a) For $\lambda=\rho^{2} / 2$ and $\rho>0$, is nonnegative and $u(0)=\rho=\|u\|_{\infty}$ such that belong to $A_{k}$ or $B_{k}$.

(b) For $\lambda \in\left(0, \rho^{2} / 2\right)$ and $\rho>0$, is sign-changing and $u(0)=\rho=\|u\|_{\infty}$ such that belong to $A_{k}$ or $B_{k}$.

(c) For $\lambda \in\left(\rho^{2} / 2, \infty\right), \lambda \neq \rho^{2}$ and $\rho>0$, is positive and $\rho_{0}=\|u\|_{\infty}$ and $u(0)=\rho$ such that belong to $C_{k}$ or $D_{k}$.

(d) For $\lambda \in(0, \infty)$ and $\rho=0$, is nonnegative and $\|u\|_{\infty}=\rho_{0}$ and $u(0)=\rho$ such that belong to $C_{k}$ or $D_{k}$.

(e) For $\lambda \in(0, \infty)$ and $\rho<0$, is sing-changing and $\|u\|_{\infty}=\rho_{0}$ and $u(0)=\rho$ such that belong to $C_{k}$ or $D_{k}$.

\section{ProOF}

Let $u$ be nontrivial solution to (1)-(2) at $\lambda$ with $u(0)=\rho$. Now multiplying (1) throughout by $u^{\prime}$ and

$$
\left[u^{\prime}\right]^{2}=2\left\{-\frac{u^{4}}{4}+\frac{\lambda u|u|}{2}+c\right\},
$$

where $\mathrm{c}$ is a constant. Applying the conditions $u(0)=\rho$ and $u^{\prime}(0)=0$, we have

$$
\left[u^{\prime}\right]^{2}=2\left\{\frac{\rho^{4}}{4}-\frac{u^{4}}{4}+\frac{\lambda}{2}(u|u|-\rho|\rho|)\right\}, \quad x \in(0,1) .
$$

Now, we define the function

$$
s \mapsto M(\rho, \lambda, s):=\frac{\rho^{4}}{4}-\frac{s^{4}}{4}+\frac{\lambda}{2}(s|s|-\rho|\rho|) \text { on } \mathbb{R},
$$

where $\lambda>0$ and $\rho \in \mathbb{R}$, are two parameters. The following lemma collects the variations of this function that follows immediately and we omit its proof.

Lemma 1 For all $\lambda \in \mathbb{R}^{+}$and $\rho \in \mathbb{R}$,

(a): $\lim _{s \rightarrow \pm \infty} M(\rho, \lambda, s)=-\infty$.

(b): The function $s \mapsto M(\rho, \lambda, s)$ is concave on $\mathbb{R}$.

(c): The function $s \mapsto M(\rho, \lambda, s)$ is increasing on $(-\infty, \sqrt{\lambda})$ and decreasing on $(\sqrt{\lambda},+\infty)$, and if $\rho>0$, then 


$$
\max _{s \in \mathbb{R}} M(\rho, \lambda, s)=M(\rho, \lambda, \sqrt{\lambda}) \begin{cases}=0, & \text { if } \lambda=\rho^{2}, \\ >0, & \text { if } \lambda \neq \rho^{2},\end{cases}
$$

if $\rho<0$, then

$$
\max _{s \in \mathbb{R}} M(\rho, \lambda, s)=M(\rho, \lambda, \sqrt{\lambda})>0
$$

if $\rho=0$, then

$$
\max _{s \in \mathbb{R}} M(\rho, \lambda, s)=M(\rho, \lambda, \sqrt{\lambda})=\frac{\lambda^{2}}{4}>0 .
$$

(d): The $y$ - intercept of the graph of $M(\rho, \lambda, \cdot)$, i.e.

$$
M(\rho, \lambda, 0)\left\{\begin{array}{l}
=0, \quad \text { if } \lambda=\frac{\rho^{2}}{2}, \rho>0, \\
>0, \quad \text { if } 0<\lambda<\frac{\rho^{2}}{2}, \rho>0, \\
<0, \quad \text { if } \frac{\rho^{2}}{2}<\lambda, \rho>0, \\
>0, \quad \text { if } \rho<0, \\
=0, \quad \text { if } \rho=0 .
\end{array}\right.
$$

(e): The function $M(\rho, \lambda, s)$ has two zeros $\rho$ and $\rho_{0}$ such that

$$
\begin{cases}\rho<\rho_{0}, & \text { if } \rho=0, \lambda \in(0, \infty), \\ \rho_{0}<\rho, & \text { if } \lambda=\frac{\rho^{2}}{2}, \rho>0, \\ \rho_{0}<0<\rho, & \text { if } 0<\lambda<\frac{\rho^{2}}{2}, \rho>0 \\ 0<\rho<\rho_{0}, & \text { if } \frac{\rho^{2}}{2}<\lambda<+\infty, \rho>0, \lambda \neq \rho^{2} \\ \rho=\rho_{0}, & \text { if } \lambda=\rho^{2}, \rho>0 \\ \rho<0<\rho_{0}, & \text { if } \rho<0\end{cases}
$$

Lemma 2 Let $u$ be a nontrivial and positive solution to (1)-(2) at $\lambda$ with $u(0)=\rho$ and $k$ be the number of interior critical points of $u$ where $k=0,1,2,3, \ldots$ and if $k>0, x_{0}$ is the first interior critical point, then

(a) The interior critical points of $u$ are $x_{0}=\frac{1}{k+1}, x_{1}=2 x_{0}, x_{2}=3 x_{0}, \cdots, x_{k-1}=$ $k x_{0}$ and

(b)

$$
\rho_{0}= \begin{cases}u\left(x_{0}\right), & \text { if } k>0 \\ u(1), & \text { if } k=0\end{cases}
$$

$$
\left.u\right|_{[0,1]}= \begin{cases}{\left[\rho_{0}, \rho\right] \text { or }\left[\rho, \rho_{0}\right],} & \text { if } \rho>0 \\ {[0, \sqrt{2 \lambda}],} & \text { if } \rho=0 \\ {\left[\rho, \rho_{0}\right],} & \text { if } \rho<0\end{cases}
$$

(c) If $u$ is decreasing at the beginning of $(0,1)$ then:

$$
\begin{gathered}
\|u\|_{\infty}=\rho=u(0)=u\left(x_{1}\right)=u\left(x_{3}\right)=\cdots, \\
\min _{x \in[0,1]} u(x)=\rho_{0}=u\left(x_{0}\right)=u\left(x_{2}\right)=u\left(x_{4}\right)=\cdots,
\end{gathered}
$$


and if $u$ is increasing at the beginning of $(0,1)$ then:

$$
\begin{gathered}
\|u\|_{\infty}=\rho_{0}=u\left(x_{0}\right)=u\left(x_{2}\right)=u\left(x_{4}\right)=\cdots, \\
\min _{x \in[0,1]} u(x)=\rho=u(0)=u\left(x_{1}\right)=u\left(x_{3}\right)=\cdots .
\end{gathered}
$$

\section{Proof of Lemma 2.}

(a) Let $x_{0}$ be the first interior critical point of $u$ and $k>0$ be the number of interior critical points of $u$. Thus the values of $u$ for any $x \in\left(0, x_{0}\right)$ must be between $u(0)=\rho$ and $u\left(x_{0}\right)$. Now we show that $u\left(x_{0}\right)=\rho_{0}$. We know that $u^{\prime}\left(x_{0}\right)=0$, hence from (3) and (4), one can conclude that $M\left(\rho, \lambda, u\left(x_{0}\right)\right)=0$, also from the Lemma $1(\mathrm{e}), M(\rho, \lambda, u(0))=0$. On the other hand $M(\rho, \lambda, u(x))>0$ for any $x \in\left(0, x_{0}\right)$. In fact, if there exists a real number $x_{00} \in\left(0, x_{0}\right)$ such that $M\left(\rho, \lambda, u\left(x_{00}\right)\right)=0$ then from (3), one can conclude that $u^{\prime}\left(x_{00}\right)=0$, i.e. $x_{00} \in\left(0, x_{0}\right)$ is an interior critical point of $u$ and this is a contradiction, because $x_{0}$ is the first interior critical point of $u$ in the interval $(0,1)$.

Now, from the Lemma 1(e), it follows that

$$
I=\left.u\right|_{\left[0, x_{0}\right]}= \begin{cases}{\left[\rho_{0}, \rho\right] \text { or }\left[\rho, \rho_{0}\right],} & \text { if } \rho>0 \\ {[0, \sqrt{2 \lambda}],} & \text { if } \rho=0 \\ {\left[\rho, \rho_{0}\right],} & \text { if } \rho<0\end{cases}
$$

Hence $u\left(x_{0}\right)=\rho_{0}$. But if $k=0$, then the values of $u$ for any $x \in(0,1)$ must be between $u(0)$ and $u(1)$. Hence by similar argument, one can show that $u(1)=\rho_{0}$.

It is clear that $x_{0}=\frac{1}{k+1}, k>0$ and also by Remark 3 , one can conclude that $2 x_{0}, 3 x_{0}, \ldots, k x_{0}$, are the rest interior critical points of $u$. The proof of part (a) follows.

(b) It easily follows from the Remark 3 and the proof of Lemma 2 (a).

(c) If $k>0, u$ must be strictly increasing or decreasing on the interval $\left(0, x_{0}\right)$. If $u$ is decreasing on $\left(0, x_{0}\right)$, then $\max _{x \in\left[0, x_{0}\right]} u(x)=$ $u(0)=\rho$ and $\min _{x \in\left[0, x_{0}\right]} u(x)=u\left(x_{0}\right)$ and by the Remark 3 and the fact that $u\left(x_{0}\right)=\rho_{0}$, one can conclude that $\max _{x \in\left[0, x_{0}\right]} u(x)=\rho=$ $u(0)=u\left(x_{1}\right)=u\left(x_{3}\right)=\cdots$, and $\min _{x \in\left[0, x_{0}\right]} u(x)=\rho_{0}=u\left(x_{0}\right)=$ $u\left(x_{2}\right)=u\left(x_{4}\right)=\cdots$. On the other hand by the Lemma 2(b) and the fact that $u$ attains its maximum and minimum values at $x=0$ and $x=x_{0}$, respectively, it follows that $\|u\|_{\infty}=u(0)=\rho$ and $\min _{x \in\left[0, x_{0}\right]} u(x)=\rho_{0}=u\left(x_{0}\right)$. Hence (5) and (6) hold. If $u$ is increasing on $\left(0, x_{0}\right)$, by similar argument, one can conclude that (7) and (8) hold. Also, if $k=0$, by similar argument, one can conclude that (5)-(8) hold. The proof of part (c) follows. $\triangle$ 
Lemma 3 Let $u$ be a nontrivial and positive solution of (1)-(2) at $\lambda \in S_{\rho}$ with $u(0)=\rho$ and $k$ be the number of interior critical points of $u$ where $k=0,1,2, \cdots$, then:

(a)

$$
S_{\rho}= \begin{cases}\left(0, \rho^{2}\right) \bigcup\left(\rho^{2},+\infty\right), & \rho>0 \\ (0, \infty), & \rho=0 \\ (0, \infty), & \rho<0\end{cases}
$$

(b) The corresponding solution is defined by

$$
\int_{\rho}^{u(x)}\{M(\rho, \lambda, s)\}^{-\frac{1}{p}} d s=\kappa\{2\}^{1 / 2} x, \quad x \in\left(0, x_{0}\right),
$$

such that

$$
\kappa= \begin{cases}-, & \text { If } \lambda \in\left(0, \rho^{2} / 2\right), \rho>0, \\ +, & \text { If } \lambda \in\left(\rho^{2} / 2,+\infty\right), \rho>0, \\ +, & \text { If } \lambda \in(0, \infty), \rho<0, \\ +, & \text { If } \lambda \in(0, \infty), \rho=0,\end{cases}
$$

and if $k>0, x_{0}$ is the first interior critical point of $u$.

Proof of Lemma 3 Let $\rho>0$. By the Lemma 2(b), $u(x) \in\left[\rho_{0}, \rho\right]$ or $\left[\rho, \rho_{0}\right]$ for any $x \in[0,1]$, and it, by the Lemma $1(\mathrm{e}),(3)$ and (4), yield that $\lambda$ must belong to $(0,+\infty)$. Now we show that, $\lambda \neq \rho^{2}$. In fact, if $\lambda=\rho^{2}$, then $\rho_{0}=\rho$ (by the Lemma $1(\mathrm{e}))$, hence by the Lemma $2(\mathrm{~b}), u \equiv \rho$ and this a contradiction, because the solution $u$ is nontrivial. Thus we conclude that $S_{\rho}=\left(0, \rho^{2}\right) \cup\left(\rho^{2},+\infty\right)$.

Also by similar argument, one can show that if $\rho \leq 0$ then $S_{p}=(0, \infty)$.

(b) Note that since every solution of (1)-(2) is symmetric about each of its interior critical points, thus it is enough to study solution on $\left[0, x_{0}\right]$ and $\left[k x_{0}, 1\right]$, where $x_{0}$ is the first interior critical point. If $\lambda \in\left(0, \rho^{2}\right)$, then by the Lemma 1(e), $\rho_{0}<\rho$ and so, by the Lemma $2(\mathrm{c}), u\left(x_{0}\right)<u(0)$. Therefore $u$ must be decreasing on $\left[0, x_{0}\right]$ and $\min _{x \in[0,1]} u(x)=\rho_{0}$. Hence from (3), we have

$$
u^{\prime}(x)=-\{2\}^{\frac{1}{2}}\{M(\rho, \lambda, u(x))\}^{\frac{1}{2}}, \quad x \in\left(0, x_{0}\right) .
$$

Also if $\lambda \in\left(\rho^{2}, \infty\right)$, then by the Lemma 1(e), $\rho_{0}>\rho$ and so, by the Lemma 2(c), $u\left(x_{0}\right)>u(0)$. Therefore $u$ must be increasing on $\left[0, x_{0}\right]$ and $\|u\|_{\infty}=\rho_{0}$. Hence from (3), we have

$$
u^{\prime}(x)=+\{2\}^{\frac{1}{2}}\{M(\rho, \lambda, u(x))\}^{\frac{1}{2}}, x \in\left(0, x_{0}\right) .
$$

Now, integrating (9) and $(10)$ on $(0, x)$ where $x \in\left(0, x_{0}\right)$, one can obtain

$$
\int_{\rho}^{u(x)}\{M(\rho, \lambda, s)\}^{-\frac{1}{2}} d s=\kappa\{2\}^{1 / 2} x, \quad x \in\left(0, x_{0}\right) .
$$

By substituting $x=x_{0}$ in (11), and using the fact that $u\left(x_{0}\right)=\rho_{0}$ (by the Lemma $2(\mathrm{c}))$, we get

$$
\int_{\Omega}\{M(\rho, \lambda, s)\}^{-\frac{1}{2}} d s=\{2\}^{1 / 2} x_{0}
$$




$$
\Omega= \begin{cases}\left(\rho_{0}, \rho\right), & \text { If } \lambda \in\left(0, \rho^{2}\right), \rho>0 \\ \left(\rho, \rho_{0}\right), & \text { If } \lambda \in\left(\rho^{2}, \infty\right), \rho>0 \\ \left(\rho, \rho_{0}\right), & \text { If } \lambda \in(0, \infty), \rho<0 \\ \left(\rho, \rho_{0}\right), & \text { If } \rho=0\end{cases}
$$

Note that in (12) the integrals are convergent. In fact,

Claim 1 The integral $\int_{\Omega}\{M(\rho, \lambda, s)\}^{-\frac{1}{2}} d s \in(0, \infty)$.

Proof of Claim 1. It is suffice to show that $\int_{\rho_{0}}^{\rho}\{M(\rho, \lambda, s)\}^{-\frac{1}{2}} d s \in$ $(0, \infty)$. For this mean, by (4) and Lemma $1(\mathrm{e})$, one can conclude that

$$
\begin{gathered}
\lim _{s \rightarrow \rho}|s-\rho|^{\frac{1}{2}}\{M(\rho, \lambda, s)\}^{-\frac{1}{2}}=\frac{1}{\left\{|\lambda| \rho\left|-\rho^{3}\right|\right\}^{\frac{1}{2}}} \in(0, \infty), \\
\lim _{s \rightarrow \rho_{0}}\left|s-\rho_{0}\right|^{\frac{1}{2}}\{M(\rho, \lambda, s)\}^{-\frac{1}{2}}=\frac{1}{\left\{|\lambda| \rho_{0}\left|-\rho_{0}^{3}\right|\right\}^{\frac{1}{2}}} \in(0, \infty) .
\end{gathered}
$$

Also we know that the integrals $\int_{\rho_{0}}^{\rho}|s-\rho|^{-\frac{1}{2}} d s$ and $\int_{\rho_{0}}^{\rho}\left|s-\rho_{0}\right|^{-\frac{1}{2}} d s$ for $p>1$, are convergent. Thus one can conclude that the convergence of the integral $\int_{\rho_{0}}^{\rho}\{M(\rho, \lambda, s)\}^{-\frac{1}{2}} d s$ is a consequence of that of the integrals $\int_{\rho_{0}}^{\rho}|s-\rho|^{-\frac{1}{2}} d s$ and $\int_{\rho_{0}}^{\rho}\left|s-\rho_{0}\right|^{-\frac{1}{2}} d s . \triangle$

Here the proof of Lemma 3 is complete. $\triangle$

Now theorem(1) follows.

Proof of theorem 2 (a) if $\rho>0$ and $\lambda=\frac{\rho^{2}}{2}$ by the Lemma $1(\mathrm{e}) \min _{x \in[0,1]} u(x)=$ $\rho_{0}<\rho$. Hence $u$ must be nonnegative solution. Also since $\rho>\rho_{0}, u$ must at first of $(0,1)$ be decreasing at the beginning of $(0,1)$. So $u$ belong to $C_{k}$ or $D_{k}$. The proof of (a) is follows. By similar argument parts (b), (c), (d) and (e) are follow. Here the proof of Theorem 2 is complete.

\section{REFERENCES}

[1] I. Addou, On the number of solutions for boundary-value problems with jumping nonlinearities, Ph.D. Thesis, Université des Sciences et de la Technologie Houari Boumedienne; Algiers, Algeria: (2000).

[2] G. A. Afruzi and M. Khaleghy Moghadaam, Existence and multiplicity results for a class of p-Laplacian problems with Neumann-Robin boundary conditions, Chaos, Solitons \& Fractals, 30 (2006) 967-973.

[3] G. A. Afrouzi and M. Khalehghy Moghaddam, Nonnegative solution Curves of Semipositone Problems With Dirichlet Boundary conditions, Nonlinear Analysis, Theory, methods, and Applications, 61 (2005) 485-489, 
[4] F. Ammar-Khodja, Une revue et quelques compléments sur la détermination du nombre des solutions de certains problèmes elliptiques semi-linéaires, Thèse Doctorat 3è Cycle, Université Pierre et Marie Curie, Paris VI, (1983).

[5] V. Anuradha, C. Maya and R. Shivaji, Positive solutions for a class of nonlinear boundary value problems with Neumann-Robin boundary conditions, J. Math. Anal. Appl. 236 (1999), $94-124$.

[6] A. Castro and R. Shivaji, Nonnegative solutions for a class non-positone problems, Proc. Roy. Soc. Edinburgh, Sect. A 108 (1988), 291-302.

[7] M. Guedda and L. Veron, Bifurcation phenomena associated to the p-Laplacian operator, Trans. Amer. Math. Soc. 310 (1988), 419-431.

[8] R. A. Khan and N. A. Asif, Positive solutions for a class of singular two point boundary value problems, J. Nonlinear Sci. Appl., 2 (2009), 126-135.

[9] A. R. Miciano and R. Shivaji, Multiple positive solutions for a class of semipositone Neumann two point boundary value problems, J. Math. Anal. Appl. 178 (1993), 102-115.

G. A. Afrouzi

Department of Mathematics, Faculty of Basic Sciences, MaZANDARAN University, BABOLSAR, IrAN

E-mail address: afrouzi@umz.ac.ir

M. Khaleghy Moghaddam

Department of Basic Sciences, Faculty of Agriculture Engineering, Sari Agricultural Sciences and Natural Resources University, Sari, Iran.

J. Mohammadpour and M. Zameni

Department of Mathematics, Islamic Azad Univercitiy

Ghaemshahr Branch, P.O. Box163, Ghaemshahr, Iran. 